\title{
GMI ablates cancer stemness and cisplatin resistance in oral carcinomas stem cells through IL-6/Stat3 signaling inhibition
}

\author{
Tung Yuan Wang ${ }^{1, *}$, Cheng-Chia Yu ${ }^{1,2,3, *}$, Pei-Ling Hsieh ${ }^{3}$, Yi-Wen Liao ${ }^{1}$, Chuan- \\ Hang $\mathrm{Yu}^{1,2}$ and Ming-Yung Chou ${ }^{1,2}$ \\ ${ }^{1}$ School of Dentistry, Chung Shan Medical University, Taichung, Taiwan \\ ${ }^{2}$ Department of Dentistry, Chung Shan Medical University Hospital, Taichung, Taiwan \\ ${ }^{3}$ Institute of Oral Sciences, Chung Shan Medical University, Taichung, Taiwan \\ *These authors have contributed equally to this work \\ Correspondence to: Ming-Yung Chou, email: myc@csmu.edu.tw \\ Chuan-Hang Yu, email: tao2008@csmu.edu.tw \\ Keywords: oral squamous cell carcinomas, immunomodulatory protein GMI, cancer stem cells, chemoresistance, IL-6/Stat3 \\ signaling \\ Received: May 04, $2017 \quad$ Accepted: June 19, $2017 \quad$ Published: July 31, 2017 \\ Copyright: Wang et al. This is an open-access article distributed under the terms of the Creative Commons Attribution License 3.0 \\ (CC BY 3.0), which permits unrestricted use, distribution, and reproduction in any medium, provided the original author and source \\ are credited.
}

\section{ABSTRACT}

Cancer stem cells (CSCs) have been identified to exert tumor-initiating ability, resulting in the recurrence, metastasis and chemoresistance of oral squamous cell carcinomas. In the present study, we showed that GMI, an immunomodulatory protein from Ganoderma microsporum, induc ed a cytotoxic effect in oral carcinomas stem cells (OCSCs). Treatment of GMI dose-dependently inhibited the expression of CSC markers, including ALDH1 activity and CD44 positivity. Moreover, GMI suppressed the self-renewal property, colony formation, migration, and invasion abilities as well as potentiated chemo-sensitivity in OCSCs. Our results suggested that the tumor suppressive effect of GMI was mediated through inhibition of IL-6/Stat3 signaling pathway. Furthermore, tumor growth was reduced in mice bearing xenograft tumors after oral administration of GMI. Taken together, we demonstrated the anti-CSC effect of GMI in oral cancer and GMI may serve as a natural cisplatin adjuvant to prevent cancer recurrence.

\section{INTRODUCTION}

Head and neck squamous cell carcinoma (HNSCC) is one of the most common cancers worldwide and the majority of HNSCC is oral squamous cell carcinoma (OSCC) [1]. Although there has been substantial progress in cancer treatment, survival rates have improved only frugally over the past few decades [2]. A significant number of patients still suffer from recurrent cancer after chemotherapy [3]. It has been shown that 3-year overall survival rate of advanced OSCC patients with recurrence was less than $30 \%$ [4]. Accumulating evidence has suggested that the resistance of radiochemotherapy is attributed to the cancer stem cells (CSCs) within the heterogeneous tumor mass [5]. It has been indicated that
CSCs exhibit stemness and are highly tumorigenic, leading to the relapse, metastasis and therapeutic refractoriness of tumor [6]. Recently, several reports have revealed that treatments against CSCs improve the efficacy of chemotherapy [7]. As such, strategy that targets CSCs may be a promising approach for OSCC.

Lingzhi, Ganoderma lucidum, is a medicinal mushroom used in traditional medicine as an herbal remedy for centuries. The therapeutic properties have been reported for Lingzhi species, such as antiinflammation [8], anti-cancer [9] and immunomodulation [10]. It contains a variety of bioactive molecules for these effects, including triterpenoids, polysaccharides and fungal immunomodulatory proteins (FIPs) [11]. To date, various FIPs have been identified to show anti-tumor, 
anti-metastatic and anti-chemoresistant capacities, such as Lingzhi-8 from Ganoderma lucidum [12, 13] or FIPgts from Ganoderma tsuga [14, 15]. Currently, another FIP from Ganoderma microsporum called GMI has been shown to possess anti-cancer effect via induction of autophagy in lung cancer cells [16, 17] and have the potential against chemo-resistance [18]. Nonetheless, the role of GMI as an anti-tumor agent to treat other type of cancer has not been examined and the signal pathways involved in this process are not well understood yet.

In this study, we assessed the effect of GMI on the stemness features and cisplatin resistance in oral cancer stem cells (OCSCs). GMI significantly suppressed the expression of CSC markers, self-renewal, clonogenic, migration and invasion abilities as well as tumorigenecity in vivo. And administration of GMI enhanced the tumor sensitization to chemotherapy. Moreover, we showed that tumor inhibitory effect of GMI was via IL-6/Stat3 signaling pathway. Altogether, we demonstrated that therapeutic effect of GMI in oral cancer was through the inhibition of CSCs and GMI may act as a potential cisplatin adjuvant for OSCC.

\section{RESULTS}

\section{The cytotoxicity effect of GMI in oral cancer stem cells (OCSCs)}

We first investigated the cell survival of these two OCSCs derived from oral cancer cell lines and normal human gingival epithelioid cell line $(\mathrm{SG})$ in order to understand the cytotoxicity of GMI. We treated these cells with increasing concentrations of GMI for 24 hours followed by MTT assay. As shown in Figure 1, GMI markedly suppressed the cell viability of two OCSCs without causing toxic damage to normal cells.

\section{GMI attenuates ALDH1 activity and CD44 positivity in OCSCs}

ALDH1 enzymatic activity [19] and CD44 positivity [20] have been proven to be highly selective markers for CSCs in OSCC. Our data suggested administration of GMI significantly resulted in a concentration-dependent decrease in ALDH1 activity of both OCSCs (Figure 2A). Likewise, the expression of CD44 was gradually downregulated in both OCSCs along with the increase in GMI concentration using flow cytometry analysis (Figure 2B). These results showed that GMI eliminated the proportion of OCSCs in a dose-dependent manner.

\section{GMI represses self-renewal and oncogenicity abilities in OCSCs}

Given that CSCs exhibit highly tumor-initiating ability and are involved in metastasis and therapeutic refractoriness of cancer [6], we sought to examine the efficacy of GMI on self-renewal, colony forming, migration and invasion abilities of OCSCs. Secondary sphere formation and anchorage-independent growth assays were used to evaluate the self-renewal and colony formation capacities, respectively. A dose-dependent suppression of tumorigenic activities, including selfrenewal (Figure 3A), colony formation (Figure 3B),

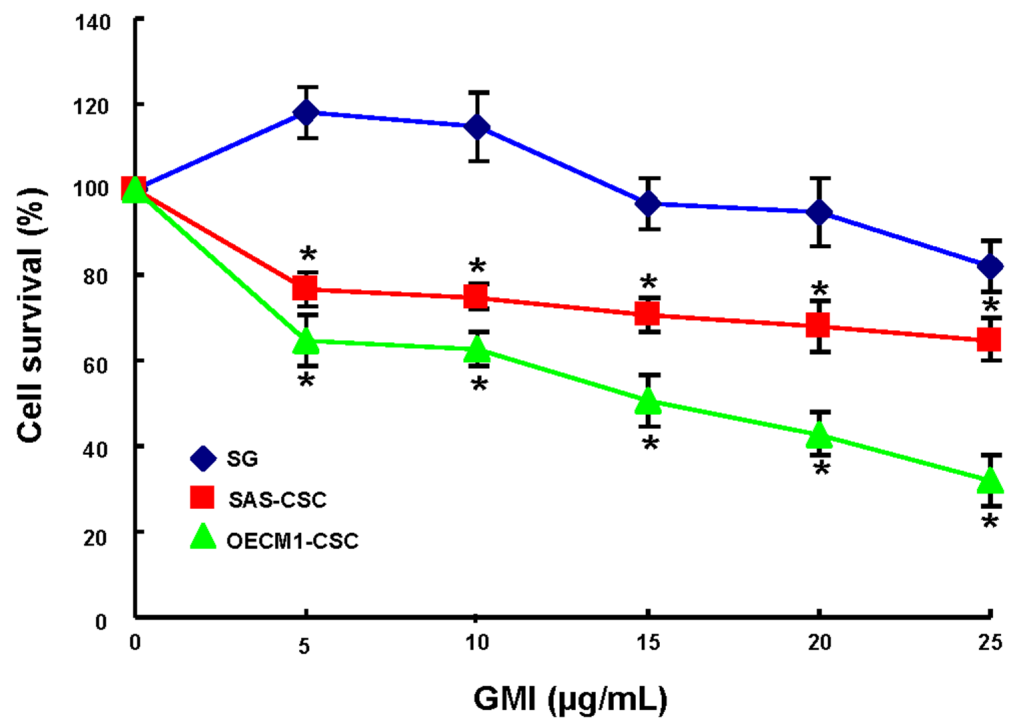

Figure 1: The cell viability effect of GMI in normal epithelial cells and OCSC. SG and OCSC were plated in wells of 96-wellplate as $1 \times 10^{4}$ cells/well in $0.1 \%$ DMSO or different concentration of recomGMI-containing medium and cultured at $37^{\circ} \mathrm{C}$ for $24 \mathrm{hr}$. Cell proliferation/survival was determined by MTT (3-(4,5-dimethylthiazol-2-yl)-2,5-diphenyl tetrazolium bromide) assay. The 570 nm absorbance of DMSO treated group was set as $100 \%$ and data were presented as percentage of DMSO control. Results are means \pm SD. *, $\mathrm{p}<0.05$ vs. Control. 
A
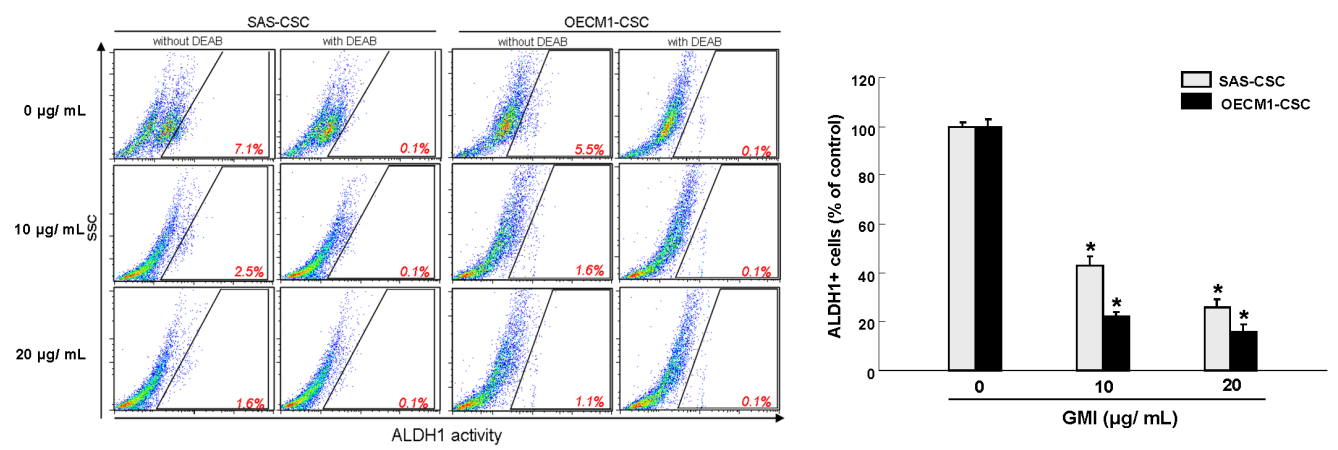

B
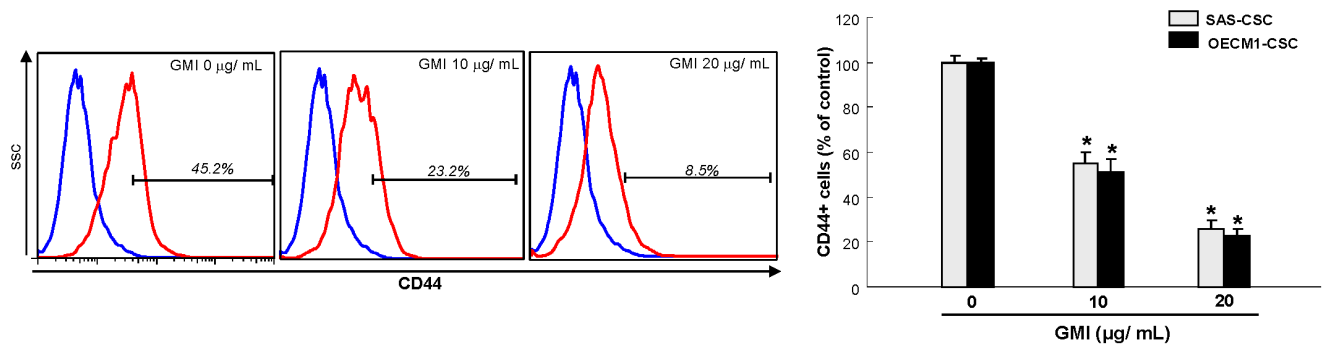

Figure 2: GMI effectively eliminates ALDH1 activity and CD44 positivity. (A) For this assay, $1 \times 10^{5}$ cells were suspended in $50 \mathrm{ml}$ of assay buffer, and ALDEFLUOR was added to the cell suspensions for a final concentration of $1 \mathrm{mM}$. For ALDH1 inhibitor control, DEAB was added to a final concentration of $150 \mathrm{mM}$. Cells were then incubated at $37^{\circ} \mathrm{C}$ for 45 min and were stained with 7-AAD on ice for $5 \mathrm{~min}$. After washing the cells with PBS, live cells (7AAD-) positive for green fluorescence were analyzed by flow cytometry (FACSCalibur ${ }^{\mathrm{TM}}$, BD Biosciences) to compare the fluorescence intensity of the DEAB-treated samples. High fluorescence was associated with high ALDH activity (ALDH+ cells). (B) Cells were stained with anti-CD44 antibody conjugated to phycoerythrin (Miltenyi Biotech., Auburn, CA, USA), with labeling according to the manufacturer's instructions. Red (>650 nm) fluorescence emission from 10,000 cells illuminated with blue $(488 \mathrm{~nm})$ excitation light was measured with a FACSCalibur (Becton Dickinson) using CellQuest software. Results are means $\pm \mathrm{SD} .{ }^{*}, \mathrm{p}<0.05$ vs. Control.

A
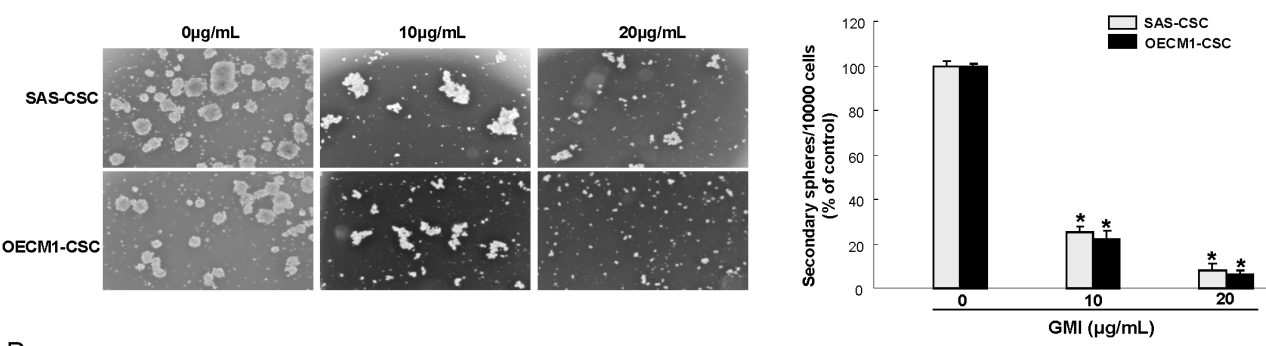

B
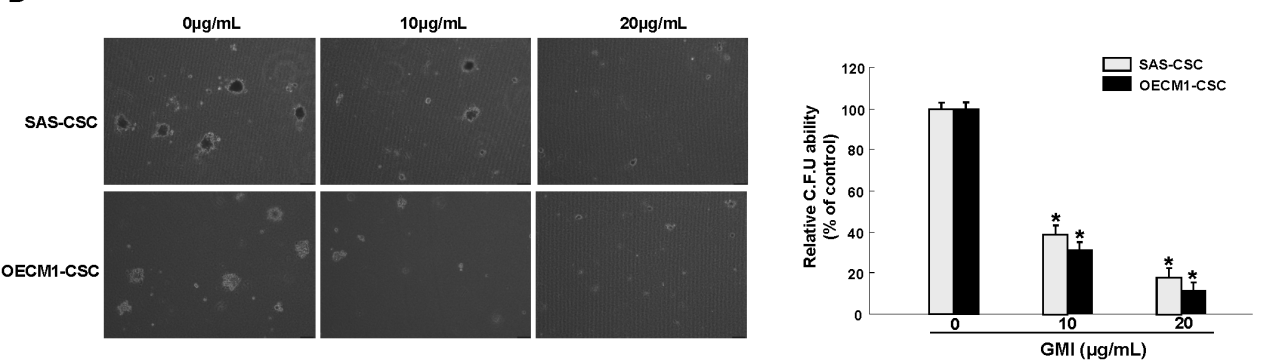

Figure 3: Inhibition of self-renewal property and clonogenicity in OCSC under GMI treatment. (A) Secondary sphere formation ability of GMI-treated cells was examined. The bar graph shows quantification of secondary sphere number. (B) OCSC with dose-dependent GMI treatment were assigned for the colony formation assay. The experiments were repeated three times and representative results were shown. Results are means \pm SD. ${ }^{*}, \mathrm{p}<0.05$. 
migration (Figure 4A), invasion (Figure 4B) properties of OCSCs was observed. Collectively, these results clearly demonstrated that GMI exerted a pronounced anti-tumor effect on OCSCs.

\section{Enhanced chemosensitivity in OCSCs by GMI}

The recurrence of cancers has been attributed to chemo-resistant CSC after conventional treatments [21], hence it is crucial to assess the chemosensitivity when evaluating the anti-tumor effect of GMI. As expected, drug-resistance was obviously more prevalent in OCSCs compared with parental OSCC cells using cell viability assay. Nevertheless, the sensitivity to Cisplatin in OCSC was dramatically improved in combination with GMI (Figure 5A). Moreover, the invasive (Figure 5B) and colony formation (Figure 5C) capacities were further abolished by Cisplatin in conjunction with GMI compared to GMI or Cisplatin alone. Overall, these findings demonstrated the enhanced anti-OCSC activity via the synergic action of GMI and Cisplatin.

\section{The anti-tumor effect of GMI is via inhibition of IL6/ Stat3 axis}

The elevated expression of IL- 6 has been found to be involved in erlotinib resistance of OSCC [22] and the immunomodulatory effect of GMI has been shown in a previous report [23]. In the current study, the expression of IL-6 in OCSCs was reduced by GMI in a dose-dependent fashion (Figure 6A). Since IL-6 is a major autocrine/ paracrine factor to activate Stat3 in OSCC [24], we examined the expression level of Stat 3 following treatment of GMI to verify the possible mechanism. Results from western blotting showed that the protein expression of phosphorylated-Stat3 was gradually down-regulated in the presence of increased GMI concentration, whereas the level of total Stat3 was not affected (Figure 6B). Furthermore, the inhibitory effect of GMI on self-renewal (Figure 6C) and invasive (Figure 6D) properties was reverted by addition of IL-6, indicating the anti-OCSCs potential of GMI was via modulation of IL-6/Stat3 axis.

\section{Administration of GMI exerts a suppressive effect on tumor growth in vivo}

To validate the anti-tumorigenic efficacy of GMI in vivo, immunocompromised mice bearing OCSC xenografts received GMI treatment or vehicle by oral gavage followed by analyses of tumor volume and expression of phosphorylated-Stat3 in tumors. As shown in Figure 7A, the tumor growth was significantly delayed after treatment of GMI. And the GMI-induced downregulation of phosphorylated-Stat3 (Figure 7B) was confirmed by western blotting.

A
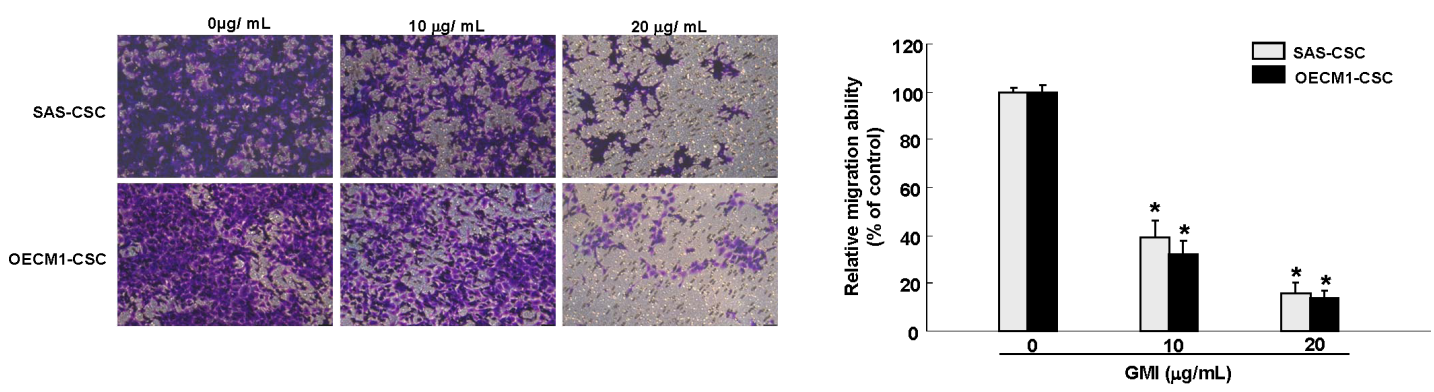

B
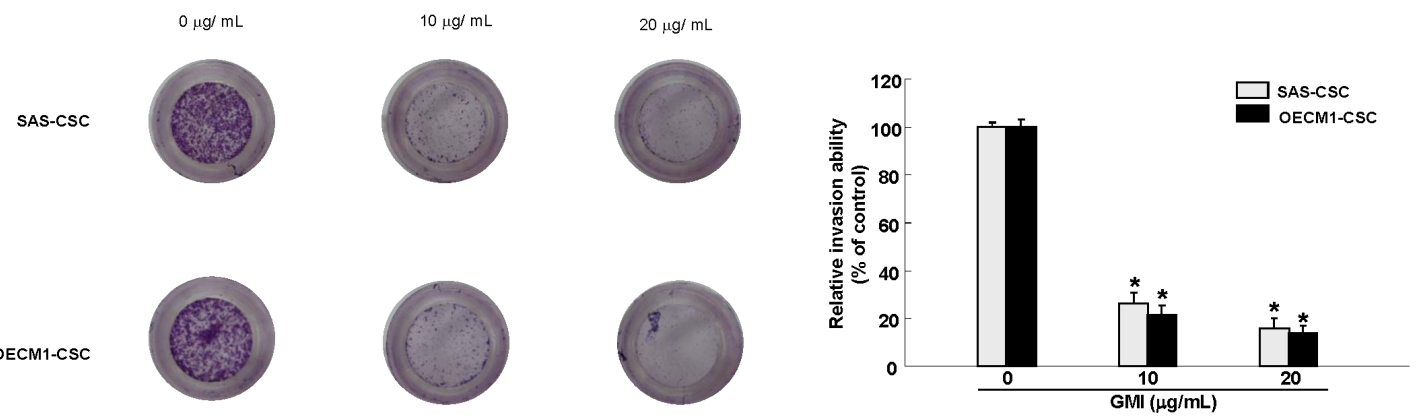

Figure 4: GMI abrogates migration and invasion capacity of OCSC. Representative images (left) and quantification (right) of (A) migration assay and (B) Matrigel invasion assay of OCSCs treated with various concentration of GMI. Experiments were performed in triplicate. Values are expressed as mean \pm SD. ${ }^{*} p<.05$ compared to control. 
A

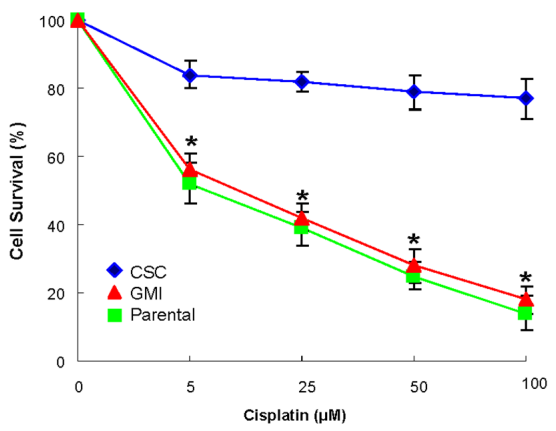

B

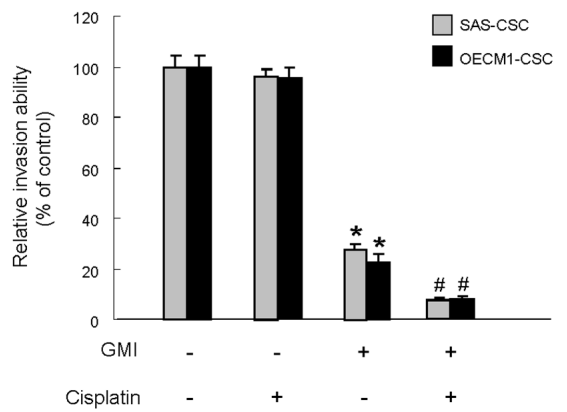

C

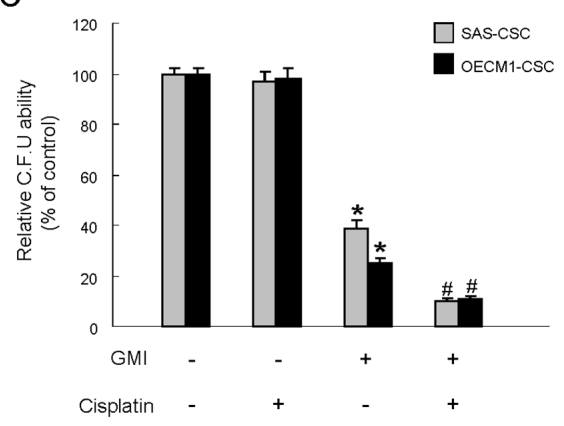

Figure 5: Enhanced sensitivity of OCSC by GMI treatment. (A) After exposure to different doses of cisplatin, the surviving cell fractions of the control or GMI-treated OCSC were evaluated. (B) Invasion ability and (C) colony-forming ability in OCSC were examined after treatment with either GMI or cisplatin treatment or both. *, p $<0.05$ GMI vs. control; \#, p $<0.05$ GMI+cisplatin vs. GMI alone.

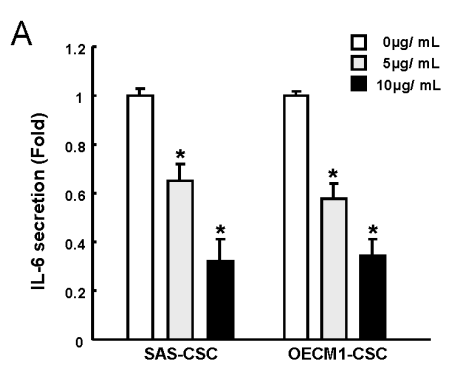

C

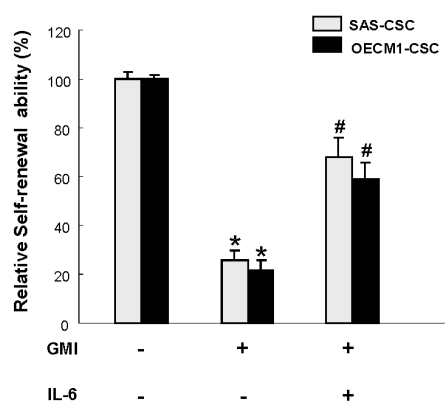

B
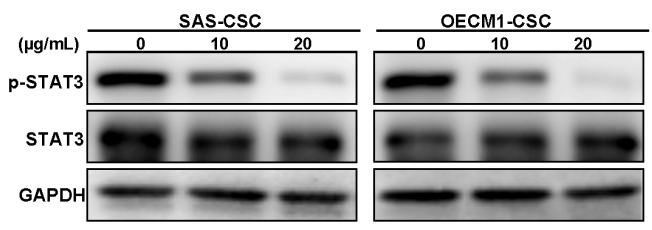

D

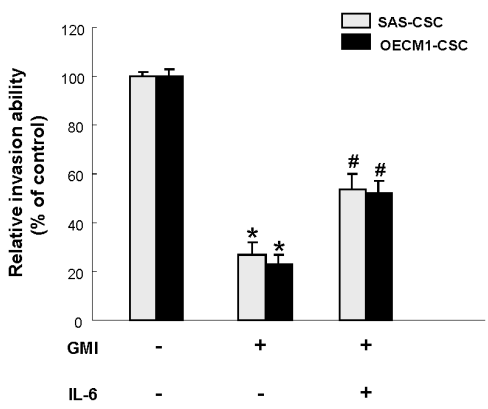

Figure 6: GMI treatment represses IL-6/STAT3 signaling of OCSC. (A) IL-6 secretion level in GMI-treated OCSC was analyzed by ELISA analysis. (B) Cell extract proteins of GMI-treated OCSC were collected and analyzed by immunoblotting against anti-p-STAT3, anti-STAT3, or anti-GAPDH antibodies as indicated. The immunoactive signal of GAPDH protein of different crude cell extracts was referred as loading control. Self-renewal (C) and invasion ability (D) in OCSC were analyzed after treatment with either GMI treatment or GMI combined IL-6 treatment. *, p $<0.05$ GMI vs control; \#, p $<0.05$ GMI+IL-6 vs. GMI alone. 


\section{DISCUSSION}

Over the past few decades, increasing attention has been paid to the use of natural products against cancer and it has been suggested that immunological approaches targeted CSCs may be a new direction of cancer therapy [25]. Previously, the effects of immunomodulatory protein GMI have been examined in various studies. It has been shown that GMI suppressed the EGF-mediated migration and invasion via blockage of PI3K/Akt pathway [17] and the TNF- $\alpha$-induced tumor invasion and inflammation through inhibition of NF- $\mathrm{KB} / \mathrm{MMP}-9$ pathway [23] in human alveolar epithelial A549 cells. Later, the anti-tumor effect of GMI in non-small cell lung cancer cells was further investigated and proved to be mediated through activation of autophagy [16]. In addition, GMI was showed to enhance Cisplatin-induced apoptosis through the inhibition of Akt/mTOR pathway $[18,26]$. These results suggested that the effects of GMI are associated with the regulation of PI3K/Akt/mTOR signaling pathway. In fact, this pathway has been shown to play a crucial role in CSC therapy [27] and it interacts with NFKB, which is integrated into essential aspects for oncogenesis $[28,29]$ and contributed to CSC phenotype [30].

In the current report, we revealed the novel antiCSCs properties of GMI and demonstrated that it potentiated the effectiveness Cisplatin-based therapy for OSCC treatment. Our findings suggested that the tumor suppressive effect of GMI was via inhibition of IL-6/Stat3 signaling. Various studies have shown that hyperactivation of Stat3 is implicated in tumor progression and treatment resistance [31,32] and Stat3 blockade enhances the efficacy of chemotherapeutic agents in OSCC $[33,34]$. Stats can be activated by a variety of signal transduction

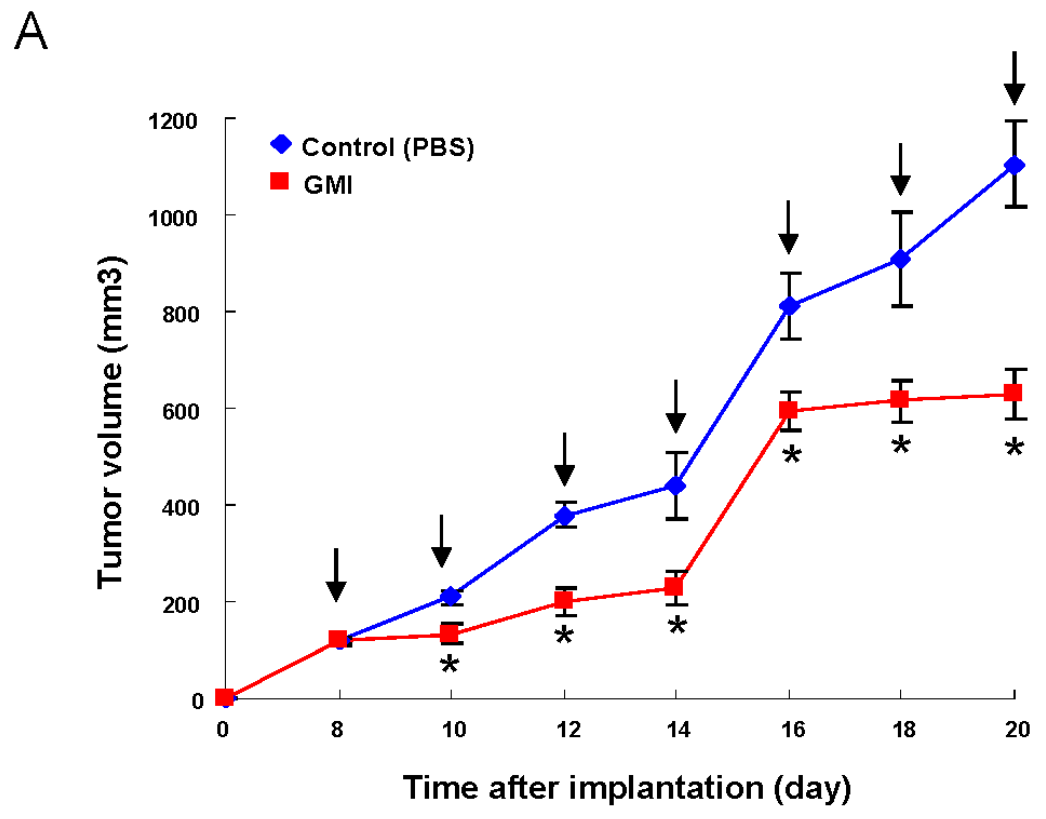

B

\begin{tabular}{|c|c|c|c|c|c|c|c|c|c|c|c|c|}
\hline & \multicolumn{2}{|c|}{$\# 1$} & \multicolumn{2}{|c|}{ \#2 } & \multicolumn{2}{|c|}{$\# 3$} & \multicolumn{2}{|c|}{$\# 4$} & \multicolumn{2}{|c|}{$\# 5$} & \multicolumn{2}{|c|}{ \#6 } \\
\hline & Ctrl. & GMI & Ctrl. & GMI & Ctrl. & GMI & Ctrl. & GMI & Ctrl. & GMI & Ctrl. & GMI \\
\hline p-STAT3 & $m$ & & 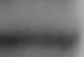 & & $-\infty$ & & 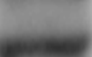 & & $=$ & & 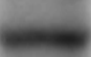 & \\
\hline GAPDH & 0 & sines & & 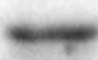 & 0 & & 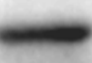 & $=$ & 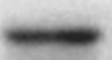 & 0 & 0 & 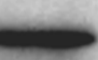 \\
\hline
\end{tabular}

Figure 7: Therapeutic delivery of GMI in OCSC-transplanted mice attenuates tumor progression in vivo. (A) After subcutaneous implantation of OCSC, BALB/c nude mice ( $N=6$ for each group) were oral-feeding treated with $\mathrm{GMI}$ and then photographed and analyzed for the and tumor volume. (B) Mice were sacrificed, and tumor sections as indicated treatments were assessed for p-Stat3 expression by western bloting analysis. Values are expressed as mean \pm SD. ${ }^{*} p<.05$ compared to control. 
pathways, such as epidermal growth factor receptor (EGFR), nicotinic receptor and interleukin (IL) receptor pathways [32]. And it has been demonstrated that IL-6 is the major autocrine/paracrine factor for Stat3 activation in OSCC [24]. Numerous studies have shown that targeting of IL-6/STAT3 signaling inhibited CSCs in vitro and in vivo $[35,36]$. Consistent with these studies, we demonstrated that administration of GMI could serve as a therapeutic approach to inhibit IL-6/Stat3 signaling, thereby attenuating cancer stemness, invasiveness and chemo-resistance.

In conclusion, these data demonstrated the antiOCSC effect of GMI in vitro and in vivo. And the tumor suppressive effect of GMI was via regulation of IL-6/ Stat3 pathway. Furthermore, GMI was a chemosensitizing agent and could use as a Cisplatin adjuvant to reduce cancer recurrence. This work provided new insight into the utilization of GMI as an anti-cancer agent for OSCC therapy.

\section{MATERIALS AND METHODS}

\section{Reagent and cell culture}

GMI, manufactured by Mycomagic Biotechnology Co., Ltd. (Taipei, Taiwan), was generated and ameliorated from Ganoderma microsporum. The CSCs derived from OSCC cell lines SAS and OECM-1 as well as normal human gingival epithelioid cell line (SG) were cultivated as previously described [37].

\section{MTT assay}

Cell viability was determined using MTT (Sigma, St. Louis, MO) to evaluate the cytotoxicity of GMI. Cells were seeded in 24-well plates $\left(1 \times 10^{4}\right.$ cells/ well) in the presence of various concentration of GMI or vehicle at $37^{\circ} \mathrm{C}$ for 24 hours followed by incubation with MTT reagent. The blue formazan crystals of viable cells were dissolved in DMSO and then evaluated spectrophotometrically at $570 \mathrm{~nm}$. DMSO-treated group was set as $100 \%$, and data were presented as percentage of DMSO control.

\section{Flow cytometry analysis}

For cell surface marker analysis, cells were stained with anti-CD44 antibody conjugated with phycoerythrin (Miltenyi Biotech., Auburn, CA, USA). And ALDEFLUOR kit (Stem Cell Technologies, Durham, NC, USA) was used to examine the ALDH1 enzymatic activity according to manufacturer's instructions. Fluorescence emission from 10,000 cells was measured with FACSCalibur (Becton Dickinson, Mountain View, CA, USA) using CellQuest software.

\section{Secondary sphere formation assay}

Cells were dissociated and cultured in the modified DMEM/F-12 supplemented with N2 (R\&D Minneapolis, MN, USA), $10 \mathrm{ng} / \mathrm{mL}$ epidermal growth factor (Invitrogen, Carlsbad, CA, USA), $10 \mathrm{ng} / \mathrm{mL}$ basic fibroblast growth factor (Invitrogen, Carlsbad, CA, USA), and penicillin/ streptomycin at $10^{3}$ live cells/low-attachment six-well plate (Corning Inc., Corning, NY, USA). Medium was changed every other day until the secondary sphere formation was observed in about 2 weeks. Cell density/ 10,000 cells were presented as the percentage of control.

\section{Soft agarose assay}

Each well of a six-well culture dish was coated with $1 \mathrm{ml}$ of bottom agar (Sigma-Aldrich) mixture (DMEM/F-12, 15\% (v/v) FBS, 0.525\% (w/v) agar). After the bottom layer was solidified, $1 \mathrm{ml}$ of top agar-medium mixture (DMEM/F-12, 15\% (v/v) FBS, 0.3\% (w/v) agar) containing $4 \times 10^{4}$ cells was added, and the dishes were incubated at $37^{\circ} \mathrm{C}$ for 2 weeks. Plates were stained with $0.01 \%$ Crystal Violet, and then the colonies were counted.

\section{Cell invasion and migration assays}

The 24-well plate Transwell system with a polycarbonate filter membrane of $8-\mu \mathrm{m}$ pore size (Corning, United Kingdom) was employed to evaluate the migration and invasion abilities of cells. The membrane was coated with Matrigel (BD Pharmingen, NJ, USA) for invasion. The cell suspensions were seeded to the upper chamber of the Transwell insert within serum-free medium at the cell density of $5 \times 10^{4}$ and $1 \times 10^{5}$ for migration and invasion assays, respectively. The lower chamber was filled with media supplemented with $10 \%$ serum. After 24 hours of incubation, the filter membrane was stained with crystal violet (Sigma-Aldrich). The migrated and invasion cancer cells were then visualized and counted from five different visual areas of 100-fold magnification under an inverted microscope.

\section{ELISA analysis}

For detection of IL-6, cells were cultured in 6-well plates with various concentration of GMI for 24 hours. Cell supernatants were collected and centrifuged to remove dead cells. The supernatants were then analyzed by ELISA using IL-6 specific kit from eBioscience according to the manufacturer's instructions.

\section{Western blot analysis}

Cell protein extraction and immunoblotting analysis were performed as previously described [38]. Briefly, 
sample was boiled at $95^{\circ} \mathrm{C}$ for 5 minutes and separated on $10 \%$ SDS-PAGE. The proteins were transferred to Hybond-ECL nitrocellulose paper or Polyvinylidene difluoride membrane (Amersham, Arlington Heights, IL, USA). The primary antibodies used were rabbit antiStat3, anti-phospho-Stat3 (cell signaling Santa Cruz Biotechnology) and mouse anti-GAPDH (Chemicon, Temecula, CA, USA). Immunoreactive protein bands were detected by the ECL detection system (Amersham Biosciences Co., Piscataway, NJ, USA).

\section{Measurement of tumor growth in vivo}

All procedures involving animals were conducted in accordance with the institutional animal welfare guidelines of the Chung Shan Medical University. 5-6 weeks old immuno-deficient nude mice (BALB/c nu/nu mice) were used for the xenograft model. OCSC $\left(1 \times 10^{4}\right.$ cells $/ 0.2 \mathrm{~mL} /$ mouse) were injected subcutaneously into the right axilla and the day of cell implantation was designated as day 0 . Mice were randomly divided into two groups and fed with either saline (control) or GMI $(150 \mu \mathrm{g} / \mathrm{day} / \mathrm{kg})$ by oral gavage 8 days post implantation. Tumor size measurement was performed using an IVIS50 animal imaging system (Xenogen Corp.). The volume was calculated (according to the following formula: [length $\times$ width $\left.^{2}\right] / 2$ ), and then analyzed by Image-Pro Plus software. After 20 days, animals were euthanized followed by tissue excision for phosphor-Stat3 analysis.

\section{Statistical analysis}

SPSS software (version 13.0; SPSS, Inc., Chicago, IL, USA) was used for statistical analysis. The presented results are representative of three independent experiments with similar results. Statistical differences were evaluated with the Student t test, and were considered significant at $p<0.05$.

\section{ACKNOWLEDGMENTS}

This study was supported by grants from Chung Shan Medical University Hospital (CSH-2016-D-003) and Ministry of Science and Technology (MOST 105-2314-B040 -008; MOST 105-2632-B-040 -003) in Taiwan.

\section{CONFLICTS OF INTEREST}

The authors have declared no conflicts of interest.

\section{REFERENCES}

1. Choi S, Myers JN. Molecular pathogenesis of oral squamous cell carcinoma: implications for therapy. J Den Res. 2008; 87:14-32.
2. Warnakulasuriya S. Global epidemiology of oral and oropharyngeal cancer. Oral Oncol. 2009; 45:309-316.

3. Gibson MK, Forastiere AA. Reassessment of the role of induction chemotherapy for head and neck cancer. Lancet Oncol. 2006; 7:565-574.

4. Hasegawa T, Shibuya Y, Takeda D, Iwata E, Saito I, Kakei Y, Sakakibara A, Akashi M, Minamikawa T, Komori T. Prognosis of oral squamous cell carcinoma patients with level IV/V metastasis: an observational study. J Craniomaxillofac Surg. 2016; 45:145-149.

5. Morrison R, Schleicher SM, Sun Y, Niermann KJ, Kim S, Spratt DE, Chung CH, Lu B. Targeting the mechanisms of resistance to chemotherapy and radiotherapy with the cancer stem cell hypothesis. J Oncol. 2011; 2011:941876.

6. Medema JP. Cancer stem cells: the challenges ahead. Nat Cell Biol. 2013; 15:338-344.

7. Vidal SJ, Rodriguez-Bravo V, Galsky M, Cordon-Cardo C, Domingo-Domenech J. Targeting cancer stem cells to suppress acquired chemotherapy resistance. Oncogene. 2014; 33:4451-4463.

8. Elsayed EA, El Enshasy H, Wadaan MA, Aziz R. Mushrooms: a potential natural source of anti-inflammatory compounds for medical applications. Mediators Inflamm. 2014; 2014:805841.

9. Yuen JW, Gohel MD. Anticancer effects of Ganoderma lucidum: a review of scientific evidence. Nutr Cancer. 2005; 53:11-17.

10. Lin ZB, Zhang HN. Anti-tumor and immunoregulatory activities of Ganoderma lucidum and its possible mechanisms. Acta Pharmacol Sin. 2004; 25:1387-1395.

11. El Enshasy HA, Hatti-Kaul R. Mushroom immunomodulators: unique molecules with unlimited applications. Trends Biotechnol. 2013; 31:668-677.

12. Wu CT, Lin TY, Hsu HY, Sheu F, Ho CM, Chen EI. Ling Zhi-8 mediates p53-dependent growth arrest of lung cancer cells proliferation via the ribosomal protein S7-MDM2-p53 pathway. Carcinogenesis. 2011; 32:1890-1896.

13. Lin TY, Hsu HY. Ling Zhi-8 reduces lung cancer mobility and metastasis through disruption of focal adhesion and induction of MDM2-mediated Slug degradation. Cancer Lett. 2016; 375:340-348.

14. Liao CH, Hsiao YM, Sheu GT, Chang JT, Wang PH, Wu MF, Shieh GJ, Hsu CP, Ko JL. Nuclear translocation of telomerase reverse transcriptase and calcium signaling in repression of telomerase activity in human lung cancer cells by fungal immunomodulatory protein from Ganoderma tsugae. Biochem Pharmacol. 2007; 74:1541-1554.

15. Li JR, Cheng CL, Yang WJ, Yang CR, Ou YC, Wu MJ, Ko JL. FIP-gts potentiate autophagic cell death against cisplatin-resistant urothelial cancer cells. Anticancer Res. 2014; 34:2973-2983.

16. Hsin IL, Ou CC, Wu TC, Jan MS, Wu MF, Chiu LY, Lue $\mathrm{KH}$, Ko JL. GMI, an immunomodulatory protein from 
Ganoderma microsporum, induces autophagy in non-small cell lung cancer cells. Autophagy. 2011; 7:873-882.

17. Lin CH, Sheu GT, Lin YW, Yeh CS, Huang YH, Lai YC, Chang JG Ko JL. A new immunomodulatory protein from Ganoderma microsporum inhibits epidermal growth factor mediated migration and invasion in A549 lung cancer cells. Process Biochem. 2010; 45:1537-1542.

18. Hsin IL, Ou CC2, Wu MF, Jan MS, Hsiao YM, Lin CH, Ko JL. GMI, an Immunomodulatory Protein from Ganoderma microsporum, Potentiates Cisplatin-Induced Apoptosis via Autophagy in Lung Cancer Cells. Mol Pharm. 2015; 12:1534-1543.

19. Clay MR, Tabor M, Owen JH, Carey TE, Bradford CR, Wolf GT, Wicha MS, Prince ME. Single-marker identification of head and neck squamous cell carcinoma cancer stem cells with aldehyde dehydrogenase. Head Neck. 2010; 32:1195-1201.

20. Prince ME, Sivanandan R, Kaczorowski A, Wolf GT, Kaplan MJ, Dalerba P, Weissman IL, Clarke MF, Ailles LE. Identification of a subpopulation of cells with cancer stem cell properties in head and neck squamous cell carcinoma. Proc Natl Acad Sci U S A. 2007; 104:973-978.

21. Dean M, Fojo T, Bates S. Tumour stem cells and drug resistance. Nat Rev Cancer. 2005; 5:275-284.

22. Stanam A, Love-Homan L, Joseph TS, Espinosa-Cotton M, Simons AL. Upregulated interleukin-6 expression contributes to erlotinib resistance in head and neck squamous cell carcinoma. Mol Oncol. 2015; 9:1371-1383.

23. Lin CH, Hsiao YM, Ou CC, Lin YW, Chiu YL, Lue KH, Chang JG, Ko JL. GMI, a Ganoderma immunomodulatory protein, down-regulates tumor necrosis factor $\alpha$-induced expression of matrix metalloproteinase 9 via $\mathrm{NF}-\kappa \mathrm{B}$ pathway in human alveolar epithelial A549 cells. J Agric Food Chem. 2010; 58:12014-12021.

24. Sriuranpong V, Park JI, Amornphimoltham P, Patel V, Nelkin BD, Gutkind JS. Epidermal growth factor receptor-independent constitutive activation of STAT3 in head and neck squamous cell carcinoma is mediated by the autocrine/paracrine stimulation of the interleukin 6/ gp130 cytokine system. Cancer Res. 2003; 63:2948-2956.

25. Pan Q, Li Q, Liu S, Ning N, Zhang X, Xu Y, Chang AE, Wicha MS. Concise review: targeting cancer stem cells using immunologic approaches. Stem Cells. 2015; 33:2085-2092.

26. Chiu LY, Hu ME, Yang TY, Hsin IL, Ko JL, Tsai KJ, Sheu GT. Immunomodulatory protein from ganoderma microsporum induces pro-death autophagy through AktmTOR-p70S6K pathway inhibition in multidrug resistant lung cancer cells. PLoS One. 2015; 10:e0125774.

27. Xia P, Xu XY. PI3K/Akt/mTOR signaling pathway in cancer stem cells: from basic research to clinical application. Am J Cancer Res. 2015; 5:1602-1609.
28. Dan HC, Cooper MJ, Cogswell PC, Duncan JA, Ting JP, Baldwin AS. Akt-dependent regulation of NF- $\{$ kappa $\}$ B is controlled by mTOR and Raptor in association with IKK. Genes Dev. 2008; 22:1490-1500.

29. Bai D, Ueno L, Vogt PK. Akt-mediated regulation of NFkappaB and the essentialness of NFkappaB for the oncogenicity of PI3K and Akt. Int J Cancer. 2009; 125:2863-2870.

30. Rinkenbaugh AL, Baldwin AS. The NF-kB oathway and cancer stem cells. Cells. 2016; 5:E16.

31. Geiger JL, Grandis JR, Bauman JE. The STAT3 pathway as a therapeutic target in head and neck cancer: barriers and innovations. Oral Oncol. 2016; 56:84-92.

32. Mali SB. Review of STAT3 (signal transducers and activators of transcription) in head and neck cancer. Oral Oncol. 2015; 51:565-569.

33. Bu LL, Zhao ZL, Liu JF, Ma SR, Huang CF, Liu B, Zhang WF, Sun ZJ. STAT3 blockade enhances the efficacy of conventional chemotherapeutic agents by eradicating head neck stemloid cancer cell. Oncotarget. 2015; 6:4194441958. https://doi.org/10.18632/oncotarget.5986.

34. Kaliyaperumal K, Sharma AK, McDonald DG, Dhindsa JS, Yount C, Singh AK, Won JS, Singh I. S-Nitrosoglutathionemediated STAT3 regulation in efficacy of radiotherapy and cisplatin therapy in head and neck squamous cell carcinoma. Redox Biol. 2015; 6:41-50.

35. van der Zee M, Sacchetti A, Cansoy M, Joosten R, Teeuwssen M, Heijmans-Antonissen C, Ewing-Graham PC, Burger CW, Blok LJ, Fodde R. IL6/JAK1/STAT3 Signaling blockade in endometrial cancer affects the ALDHhi/ CD126+ stem-like component and reduces tumor burden. Cancer Res. 2015; 75:3608-3622.

36. Han Z, Wang X, Ma L, Chen L, Xiao M, Huang L, Cao Y, Bai J, Ma D, Zhou J, Hong Z. Inhibition of STAT3 signaling targets both tumor-initiating and differentiated cell populations in prostate cancer. Oncotarget. 2014; 5:84168428. https://doi.org/10.18632/oncotarget.2314.

37. Yu CC, Tsai LL, Wang ML, Yu CH, Lo WL, Chang YC, Chiou GY, Chou MY, Chiou SH. miR145 targets the SOX9/ ADAM17 axis to inhibit tumor-initiating cells and IL-6mediated paracrine effects in head and neck cancer. Cancer Res. 2013; 73:3425-3440.

38. Yang PY, Hsieh PL, Wang TH, Yu CC, Lu MY, Liao YW, Lee TH, Peng CY. Andrographolide impedes cancer stemness and enhances radio-sensitivity in oral carcinomas via miR-218 activation. Oncotarget. 2017; 8:4196-4207. https://doi.org/10.18632/oncotarget.13755. 